\title{
A Fast Image Restoration Algorithm Based on a Fixed Point and Optimization Method
}

\author{
Adisak Hanjing ${ }^{1}$ and Suthep Suantai ${ }^{2, *}$ \\ 1 Department of Mathematics, Faculty of Science, Chiang Mai University, Chiang Mai 50200, Thailand; \\ adisak_h@cmu.ac.th \\ 2 Data Science Research Center, Research Center in Mathematics and Applied Mathematics, Department of \\ Mathematics, Faculty of Science, Chiang Mai University, Chiang Mai 50200, Thailand \\ * Correspondence: suthep.s@cmu.ac.th
}

Received: 19 February 2020; Accepted: 6 March 2020; Published: 8 March 2020

check for updates

\begin{abstract}
In this paper, a new accelerated fixed point algorithm for solving a common fixed point of a family of nonexpansive operators is introduced and studied, and then a weak convergence result and the convergence behavior of the proposed method is proven and discussed. Using our main result, we obtain a new accelerated image restoration algorithm, called the forward-backward modified W-algorithm (FBMWA), for solving a minimization problem in the form of the sum of two proper lower semi-continuous and convex functions. As applications, we apply the FBMWA algorithm to solving image restoration problems. We analyze and compare convergence behavior of our method with the others for deblurring the image. We found that our algorithm has a higher efficiency than the others in the literature.
\end{abstract}

Keywords: Hilbert space; proximal methods; fixed point algorithm; forward-backward algorithm; image restoration problem

\section{Introduction}

It is well-known that fixed point theory has relevant applications in many branches of analysis [1-9] and it can be applied to solving many areas of science and applied science, engineering, economics and medicine, such as image/signal processing [10-17] and modeling intensity modulated radiation theory treatment planning [18-20]. Many real life problems can be equivalently formulated as fixed point problems, meaning that one has to find a fixed point of some operators. One of most popular fixed point algorithms is Picard iteration. Up to now, many fixed point algorithms have been introduced and studied to solve various kinds of real world problems, such as Mann iteration [7], Ishikawa iteration [4], SP-iteration [21] and W-iteration [22].

The image restoration problem is an important topic in image processing. This problem can be transformed to an optimization problem using the least absolute shrinkage and selection operator (LASSO) model. There are several optimization and fixed point methods for such problem; see [23-27] for examples. One of the most popular methods for solving the image restoration problem is FISTA (fast iterative shrinkage-thresholding algorithm). This method was shown by Beck and Teboulle in [28] to have more efficiency than the previous methods in the literature.

In this paper, we focus our attention on a new accelerated algorithm that has been developed from the view of fixed point. For instance, Wongyai and Suantai [22] proposed the W-algorithm for solving a fixed point problem of a continuous function, and proved that the $\mathrm{W}$-algorithm has a convergence rate better than the others. Motivated by this idea, we propose a new algorithm by modification of $\mathrm{W}$-algorithm for solving a common fixed point problem of a countable family of nonexpansive operators. We also prove the convergence of our algorithm under some conditions and apply it to 
solving the image restoration problem and compare its efficiency with other methods in term of PSNR (peak signal-to-noise ratio).

The organization of this paper is as follows. In Section 2, we briefly describe background and related algorithms in the literature. In Section 3, we describe some notation and useful lemmas for the latter section. In Section 4, we introduce our proposed algorithm for the common fixed point problem, giving the theoretical proofs of its convergence under particular conditions. In Section 5, we apply our algorithm to solving the image restoration problem and compare its performance with other existing methods. Finally, we conclude our work in Section 6.

\section{Background and Related Algorithms}

In this section, we recall the background of a mathematical model for the image restoration problem and some related algorithms used to solving this problem. A simple model for image restoration problem is formulated by the linear model:

$$
A y=a+v,
$$

where $y \in \mathbb{R}^{n \times 1}$ is an original image, $a \in \mathbb{R}^{m \times 1}$ is the observed image, $v$ is additive noise and $A \in \mathbb{R}^{m \times n}$ is the blurring operation. In order to solve the problem (1), Tibshirani in [29], introduced the least absolute shrinkage and selection operator (LASSO) for solving the following minimization problem:

$$
\min _{y}\left\{\|A y-a\|_{2}^{2}+\lambda\|y\|_{1}\right\}
$$

where $\lambda>0$ is a regularization parameter, $\|y\|_{1}=\sum_{i=1}^{n}\left|y_{i}\right|$, and $\|y\|_{2}=\sqrt{\sum_{i=1}^{n}\left|y_{i}\right|^{2}}$. The general minimization problem which includes (2) as a special case is the following minimization problem:

$$
\min _{y \in \mathbb{R}^{n}}\{F(y):=f(y)+h(y)\},
$$

where $h: \mathbb{R}^{n} \rightarrow \mathbb{R} \cup\{+\infty\}$ is proper convex and lower semi-continuous, and $f: \mathbb{R}^{n} \rightarrow \mathbb{R}$ is a convex and differentiable function such that $\nabla f$ is a Lipschitz continuity with constant $L>0$. The set of minimizers of $F$ is denoted by $\operatorname{Argmin}(F)$.

The classical forward-backward splitting (FBS) algorithm [30] for problem (3) is given by the following iterative formula:

$$
x_{n+1}=\underbrace{\operatorname{prox}_{c_{n} h}}_{\text {backward step }} \underbrace{\left(I-c_{n} \nabla f\right)}_{\text {forward step }}\left(x_{n}\right), \quad c_{n} \in(0,2 / L), n \in \mathbb{N},
$$

where $x_{1} \in \mathbb{R}^{n}, c_{n}$ is the step-size, $I$ is an identity operator and prox $_{h}$ is the proximity operator of $h$ defined by $\operatorname{prox}_{h}(x):=\underset{y}{\operatorname{argmin}}\left\{h(y)+\frac{1}{2}\|x-y\|_{2}^{2}\right\}$; see [31] for more details. In different literature, FBS is also called the iterative denoising method [32], Landweber iteration [33] or the fixed point continuation (FPC) algorithm [34]. In the last several years, some acceleration techniques have been proposed in order to accelerate the convergence rate of the studied algorithms.

The inertial forward-backward splitting (IFBS) was proposed by Moudafi and Oliny in [35] as follows:

$$
\begin{aligned}
& y_{n}=x_{n}+\theta_{n}\left(x_{n}-x_{n-1}\right), \\
& x_{n+1}=\operatorname{prox}_{c_{n} h}\left(y_{n}-c_{n} \nabla f\left(x_{n}\right)\right), \quad c_{n} \in(0,2 / L), \quad n \in \mathbb{N},
\end{aligned}
$$

where $x_{0}, x_{1} \in \mathbb{R}^{n}, \theta_{n}$ is the inertial parameter which controls the momentum $x_{n}-x_{n-1}$. The convergence of IFBS can be guaranteed under proper choices of $c_{n}$ and $\theta_{n}$. 
The fast iterative shrinkage-thresholding algorithm (FISTA) was proposed by Beck and Teboulle in [28] as follows:

$$
\left\{\begin{array}{l}
y_{n}=\operatorname{prox}_{\frac{1}{L} h}\left(x_{n}-\frac{1}{L} \nabla f\left(x_{n}\right)\right), \\
t_{n+1}=\frac{1+\sqrt{1+4 t_{n}^{2}}}{2}, \quad \theta_{n}=\frac{t_{n}-1}{t_{n+1}}, \\
x_{n+1}=y_{n}+\theta_{n}\left(y_{n}-y_{n-1}\right), \quad n \in \mathbb{N},
\end{array}\right.
$$

where $x_{1}=y_{0} \in \mathbb{R}^{n}, t_{1}=1$. They proved the convergence rate of the FISTA and applied the FISTA to image restoration problem. Very recently, Liang and Schonlieb [36] modified FISTA by replacing $t_{n+1}=\left(p+\sqrt{q+r t_{n}^{2}}\right) / 2$ where $p, q>0$ and $0<r \leq 4$, and proved the weak convergence theorem of FISTA.

The new accelerated proximal gradient algorithm (NAGA) was proposed by Verma and Shukla in [37] as follows:

$$
\begin{aligned}
& y_{n}=x_{n}+\theta_{n}\left(x_{n}-x_{n-1}\right), \\
& x_{n+1}=T_{n}\left[\left(1-\alpha_{n}\right) y_{n}+\alpha_{n} T_{n} y_{n}\right], \quad n \in \mathbb{N},
\end{aligned}
$$

where $x_{0}, x_{1} \in \mathbb{R}^{n}, T_{n}$ is the forward-backward operator of $f$ and $h$ with respect to $c_{n} \in(0,2 / L)$. They proved the convergence and stability of the algorithm under a few specific conditions, and applied the algorithm for solving the convex minimization problem with sparsity-inducing regularizes for multitask learning framework.

\section{Preliminaries}

Let us review some important definitions and useful lemmas needed for the convergence theorem presented in the next section.

Let $H$ be a real Hilbert space with norm $\|\cdot\|$ and inner product $\langle\cdot, \cdot\rangle$, and $C$ be a nonempty closed convex subset of $H$. A mapping $T: C \rightarrow C$ is said to be a L-Lipschitz operator if there exists $L>0$ such that $\|T x-T y\| \leq L\|x-y\|$ for all $x, y \in C$. An $L$-Lipschitz operator is called a nonexpansive operator if $L=1$. The set of all fixed points of $T$ is denoted by $\operatorname{Fix}(T)$; i.e., Fix $(T):=\{x \in C: T x=x\}$. Let $\left\{T_{n}\right\}$ and $\Omega$ be families of nonexpansive operators of $C$ into itself such that $\varnothing \neq$ Fix $(\Omega) \subset \Gamma:=$ $\cap_{n=1}^{\infty}$ Fix $\left(T_{n}\right)$, where Fix $(\Omega)$ is the set of all common fixed points of $\Omega$, and let $\omega_{w}\left(x_{n}\right)$ denote the set of all weak-cluster points of a bounded sequence $\left\{x_{n}\right\}$ in $C$. A sequence $\left\{T_{n}\right\}$ is said to satisfy the NST (Nakajo, Shimoji and Takahashi1)-condition (I) with $\Omega$ [38], if for every bounded sequence $\left\{x_{n}\right\}$ in $C$,

$$
\lim _{n \rightarrow+\infty}\left\|x_{n}-T_{n} x_{n}\right\|=0 \Longrightarrow \lim _{n \rightarrow+\infty}\left\|x_{n}-T x_{n}\right\|=0 \forall T \in \Omega \text {. }
$$

If $\Omega$ is singleton, i.e., $\Omega=T$, then $\left\{T_{n}\right\}$ is said to satisfy the NST-condition (I) with $T$. After that, Nakajo et al. [39] introduced the NST* condition which is more general than that of NST-condition. A sequence $\left\{T_{n}\right\}$ is said to satisfy the NST*-condition if for every bounded sequence $\left\{x_{n}\right\}$ in $C$,

$$
\lim _{n \rightarrow+\infty}\left\|x_{n}-T_{n} x_{n}\right\|=\lim _{n \rightarrow+\infty}\left\|x_{n}-x_{n+1}\right\|=0 \Longrightarrow \omega_{w}\left(x_{n}\right) \subset \Gamma .
$$

It follows directly from above definition that if $\left\{T_{n}\right\}$ satisfies the NST-condition (I), then $\left\{T_{n}\right\}$ satisfies the $\mathrm{NST}^{*}$-condition. Observe that if $h: H \rightarrow \mathbb{R} \cup\{+\infty\}$ is a proper, convex and lower semicontinuous function, then for all $x \in H$ the $\operatorname{prox}_{h}(x)$ exists and is unique; cf. [40]. It is well-known that

$$
x^{*} \in \operatorname{Argmin}(f+h) \Longleftrightarrow 0 \in \partial h\left(x^{*}\right)+\nabla f\left(x^{*}\right),
$$

where $\partial h$ is the subdifferential of $h$ defined by $\partial h\left(x^{*}\right):=\left\{u: h(x) \geq\left\langle u, x-x^{*}\right\rangle+h\left(x^{*}\right) \forall x\right\}$ and $\nabla f$ is the gradient of $f$; see [41] for more details. 
Note that the subdifferential operator $\partial h$ is a maximal monotone (see [42] for more details) and the solution of (3) is a fixed point of the following operator:

$$
x^{*} \in \operatorname{Argmin}(f+h) \Longleftrightarrow x^{*}=J_{c \partial h}(I-c \nabla f)\left(x^{*}\right)=\operatorname{prox}_{c h}(I-c \nabla f)\left(x^{*}\right),
$$

where $c>0$ and $J_{\partial h}$ is the resolvent of $\partial h$ defined by $J_{\partial h}=(I+\partial h)^{-1}$. If $c \in\left(0, \frac{2}{L}\right)$, we know that $\operatorname{prox}_{c h}(I-c \nabla f)$ is a nonexpansive mapping. The operator $\operatorname{prox}_{c h}(I-c \nabla f)$ is known as the forward-backward operator of $f$ and $h$ with respect to $c$. We end this part with the following lemmas which will be used to prove our main results.

Lemma 1 ([43]). For a real Hilbert space $H$, let $h: H \rightarrow \mathbb{R} \cup\{+\infty\}$ be proper convex and lower semi-continuous function, and $f: H \rightarrow \mathbb{R}$ be convex differentiable with gradient $\nabla f$ being L-Lipschitz constant for some $L>0$. If $\left\{T_{n}\right\}$ is the forward-backward operator of $f$ and $h$ with respect to $c_{n} \in(0,2 / L)$ such that $c_{n}$ converges to $c$, then $\left\{T_{n}\right\}$ satisfies NST-condition (I) with $T$, where $T$ is the forward-backward operator of $f$ and $h$ with respect to $c \in(0,2 / L)$.

Lemma 2 ([44]). Let H be a real Hilbert space. Then the following results hold:

(i) $\quad\|t u+(1-t) v\|^{2}=t\|u\|^{2}+(1-t)\|v\|^{2}-t(1-t)\|u-v\|^{2} \quad \forall t \in[0,1], \forall u, v \in H$;

(ii) $\quad\|u \pm v\|^{2}=\|u\|^{2} \pm 2\langle u, v\rangle+\|v\|^{2} \quad \forall u, v \in H$.

Lemma 3 ([45]). Let $\left\{a_{n}\right\},\left\{b_{n}\right\}$ and $\left\{\gamma_{n}\right\}$ be sequences of nonnegative real numbers such that $a_{n+1} \leq$ $\left(1+\gamma_{n}\right) a_{n}+b_{n}, n \in \mathbb{N}$. If $\sum_{n=1}^{\infty} \gamma_{n}<+\infty$ and $\sum_{n=1}^{\infty} b_{n}<+\infty$, then $\lim _{n \rightarrow+\infty} a_{n}$ exists.

Lemma 4 (Opial [46]). Let $H$ be a Hilbert space and $\left\{x_{n}\right\}$ be a sequence in $H$ such that there exists a nonempty set $\Gamma \subset H$ satisfying

(i) For every $p \in \Gamma, \lim _{n \rightarrow+\infty}\left\|x_{n}-p\right\|$ exists;

(ii) Each weak-cluster point of the sequence $\left\{x_{n}\right\}$ is in $\Gamma$.

Then there exists $x^{*} \in \Gamma$ such that $\left\{x_{n}\right\}$ weakly converges to $x^{*}$.

\section{Main Results}

In this section, we propose a modified W-algorithm which is called "MWA" for finding a common fixed point of a countable family of nonexpansive operators in a real Hilbert space. We are now ready to introduce the MWA algorithm by assuming the following:

- $\quad H$ is a real Hilbert space;

- $\left\{T_{n}: H \rightarrow H\right\}$ is a family of nonexpansive operators;

- $\quad\left\{T_{n}\right\}$ satisfies the NST*-condition;

- $\quad \Gamma:=\cap_{n=1}^{\infty} \operatorname{Fix}\left(T_{n}\right) \neq \varnothing$.

We aim to prove a weak convergence theorem of Algorithm 1 (MWA) to a common fixed point of $T_{n}$. We start with the following supporting lemma.

Lemma 5. Let $\left\{a_{n}\right\}$ and $\left\{\theta_{n}\right\}$ be sequences of nonnegative real numbers such that

$$
a_{n+1} \leq\left(1+\theta_{n}\right) a_{n}+\theta_{n} a_{n-1}, \quad n \in \mathbb{N} .
$$

Then the following holds

$$
a_{n+1} \leq K \cdot \prod_{j=1}^{n}\left(1+2 \theta_{j}\right), \quad \text { where } K=\max \left\{a_{1}, a_{2}\right\} .
$$

Moreover, if $\sum_{n=1}^{\infty} \theta_{n}<+\infty$, then $\left\{a_{n}\right\}$ is bounded. 
Proof. Given $K=\max \left\{a_{1}, a_{2}\right\}$, we have

$$
\begin{aligned}
a_{3} & \leq\left(1+2 \theta_{2}\right) K \\
a_{4} & \leq\left(1+\theta_{3}\right) a_{3}+\theta_{3} a_{2} \leq\left(1+\theta_{3}\right)\left(1+2 \theta_{2}\right) K+\theta_{3} K \\
& \leq\left(1+\theta_{3}\right)\left(1+2 \theta_{2}\right) K+\theta_{3}\left(1+2 \theta_{2}\right) K=\left(1+2 \theta_{3}\right)\left(1+2 \theta_{2}\right) K .
\end{aligned}
$$

By Mathematical induction, we obtain

$$
a_{n+1} \leq K \cdot \prod_{j=2}^{n}\left(1+2 \theta_{j}\right) \leq K \cdot \prod_{j=1}^{n}\left(1+2 \theta_{j}\right) .
$$

Note that the infinite product of the $\left(1+2 \theta_{j}\right)$ converges if the infinite sum of the $\theta_{j}$ converges. Indeed, $\left\{a_{n}\right\}$ is bounded if $\sum_{n=1}^{\infty} \theta_{n}<+\infty$.

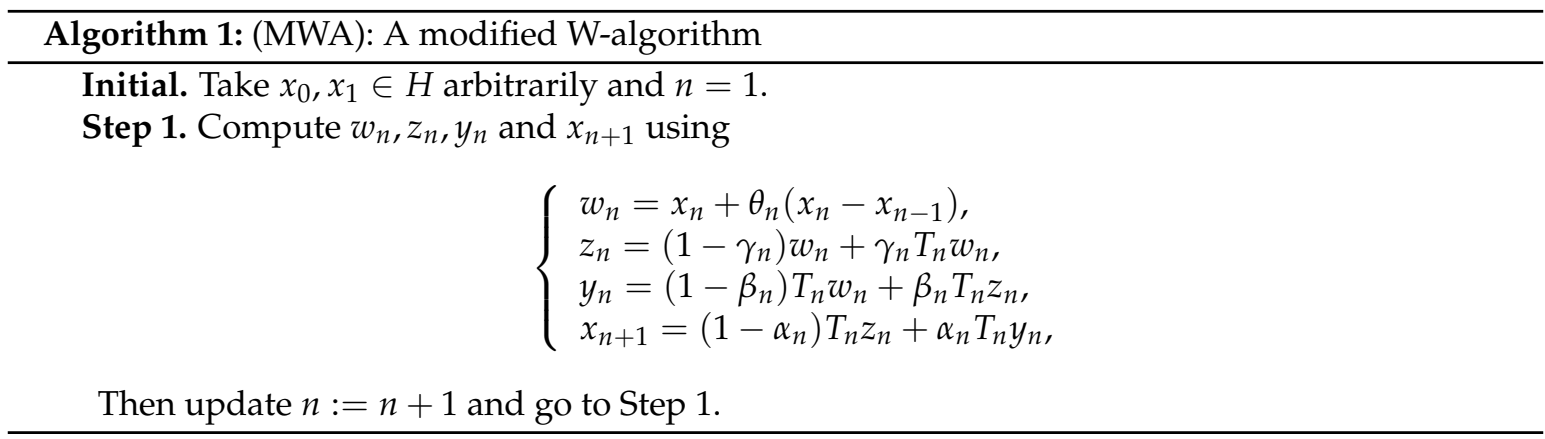

Now, we present the main convergence result of Algorithm 1 (MWA) under some suitable control conditions.

Theorem 6. Let $\left\{x_{n}\right\}$ be a sequence generated by Algorithm 1 (MWA) where $\gamma_{n} \in\left[a_{1}, b_{1}\right] \subset(0,1), \beta_{n} \in$ $[0,1], \alpha_{n} \in\left[0, b_{2}\right] \subset[0,1), \theta_{n} \geq 0$ and $\sum_{n=1}^{\infty} \theta_{n}<+\infty$. Then the following hold:

(i) $\left\|x_{n+1}-x^{*}\right\| \leq K \cdot \prod_{j=1}^{n}\left(1+2 \theta_{j}\right)$, where $K=\max \left\{\left\|x_{1}-x^{*}\right\|,\left\|x_{2}-x^{*}\right\|\right\}$ and $x^{*} \in \Gamma$.

(ii) $\left\{x_{n}\right\}$ converges weakly to a point in $\Gamma$.

Proof. (i) Let $x^{*} \in \Gamma$. By Algorithm 1, we have

$$
\begin{aligned}
\left\|w_{n}-x^{*}\right\| & \leq\left\|x_{n}-x^{*}\right\|+\theta_{n}\left\|x_{n}-x_{n-1}\right\|, \\
\left\|z_{n}-x^{*}\right\| & \leq\left(1-\gamma_{n}\right)\left\|w_{n}-x^{*}\right\|+\gamma_{n}\left\|T_{n} w_{n}-x^{*}\right\| \leq\left\|w_{n}-x^{*}\right\|, \\
\left\|y_{n}-x^{*}\right\| & \leq\left(1-\beta_{n}\right)\left\|T_{n} w_{n}-x^{*}\right\|+\beta_{n}\left\|T_{n} z_{n}-x^{*}\right\| \\
& \leq\left(1-\beta_{n}\right)\left\|w_{n}-x^{*}\right\|+\beta_{n}\left\|z_{n}-x^{*}\right\| \\
& \leq\left\|w_{n}-x^{*}\right\|,
\end{aligned}
$$

and

$$
\begin{aligned}
\left\|x_{n+1}-x^{*}\right\| & \leq\left(1-\alpha_{n}\right)\left\|T_{n} z_{n}-x^{*}\right\|+\alpha_{n}\left\|T_{n} y_{n}-x^{*}\right\| \\
& \leq\left(1-\alpha_{n}\right)\left\|z_{n}-x^{*}\right\|+\alpha_{n}\left\|y_{n}-x^{*}\right\| \\
& \leq\left\|w_{n}-x^{*}\right\| .
\end{aligned}
$$


From (10) and (13), we get

$$
\left\|x_{n+1}-x^{*}\right\| \leq\left\|x_{n}-x^{*}\right\|+\theta_{n}\left\|x_{n}-x_{n-1}\right\| .
$$

This implies

$$
\left\|x_{n+1}-x^{*}\right\| \leq\left(1+\theta_{n}\right)\left\|x_{n}-x^{*}\right\|+\theta_{n}\left\|x_{n-1}-x^{*}\right\| .
$$

Apply Lemma 5, we get $\left\|x_{n+1}-x^{*}\right\| \leq K \cdot \prod_{j=1}^{n}\left(1+2 \theta_{j}\right)$, where $K=\max \left\{\left\|x_{1}-x^{*}\right\|,\left\|x_{2}-x^{*}\right\|\right\}$. Since $\sum_{n=1}^{\infty} \theta_{n}<+\infty$, it follows that $\left\{x_{n}\right\}$ is bounded. This implies $\sum_{n=1}^{\infty} \theta_{n}\left\|x_{n}-x_{n-1}\right\|<+\infty$.

(ii) By (14) and Lemma 3, we obtain that $\lim _{n \rightarrow+\infty}\left\|x_{n}-x^{*}\right\|$ exists. By Lemma 2(ii), we obtain

$$
\left\|w_{n}-x^{*}\right\|^{2} \leq\left\|x_{n}-x^{*}\right\|^{2}+\theta_{n}^{2}\left\|x_{n}-x_{n-1}\right\|^{2}+2 \theta_{n}\left\|x_{n}-x^{*}\right\|\left\|x_{n}-x_{n-1}\right\| .
$$

By Lemma 2(i), we obtain

$$
\begin{aligned}
\left\|z_{n}-x^{*}\right\|^{2} & =\left(1-\gamma_{n}\right)\left\|w_{n}-x^{*}\right\|^{2}+\gamma_{n}\left\|T_{n} w_{n}-x^{*}\right\|^{2}-\gamma_{n}\left(1-\gamma_{n}\right)\left\|w_{n}-T_{n} w_{n}\right\|^{2} \\
& \leq\left\|w_{n}-x^{*}\right\|^{2}-\gamma_{n}\left(1-\gamma_{n}\right)\left\|w_{n}-T_{n} w_{n}\right\|^{2} .
\end{aligned}
$$

Using Lemma 2(i) agai,n together with (12) and (17), we get

$$
\begin{aligned}
\left\|x_{n+1}-x^{*}\right\|^{2} \leq & \left(1-\alpha_{n}\right)\left\|T_{n} z_{n}-x^{*}\right\|^{2}+\alpha_{n}\left\|T_{n} y_{n}-x^{*}\right\|^{2} \\
\leq & \left(1-\alpha_{n}\right)\left\|z_{n}-x^{*}\right\|^{2}+\alpha_{n}\left\|y_{n}-x^{*}\right\|^{2} \\
\leq & \left\|w_{n}-x^{*}\right\|^{2}-\left(1-\alpha_{n}\right) \gamma_{n}\left(1-\gamma_{n}\right)\left\|w_{n}-T_{n} w_{n}\right\|^{2} \\
\leq & \left\|x_{n}-x^{*}\right\|^{2}+\theta_{n}^{2}\left\|x_{n}-x_{n-1}\right\|^{2}+2 \theta_{n}\left\|x_{n}-x^{*}\right\|\left\|x_{n}-x_{n-1}\right\| \\
& -\left(1-\alpha_{n}\right) \gamma_{n}\left(1-\gamma_{n}\right)\left\|w_{n}-T_{n} w_{n}\right\|^{2} .
\end{aligned}
$$

Since $\sum_{n=1}^{\infty} \theta_{n}\left\|x_{n}-x_{n-1}\right\|<+\infty$ and $\lim _{n \rightarrow+\infty}\left\|x_{n}-x^{*}\right\|$ exists, it follows that $\lim _{n \rightarrow+\infty} \| w_{n}-$ $T_{n} w_{n} \|=0$. Note that

$$
\left\|x_{n}-T_{n} x_{n}\right\| \leq\left\|x_{n}-w_{n}\right\|+\left\|w_{n}-T_{n} w_{n}\right\|+\left\|T_{n} w_{n}-T_{n} x_{n}\right\| \leq 2\left\|x_{n}-w_{n}\right\|+\left\|w_{n}-T_{n} w_{n}\right\|,
$$

and

$$
\begin{aligned}
\left\|y_{n}-z_{n}\right\| & \leq\left\|y_{n}-w_{n}\right\|+\left\|w_{n}-z_{n}\right\| \\
& \leq\left\|T_{n} w_{n}-w_{n}\right\|+\beta_{n}\left\|T_{n} z_{n}-T_{n} w_{n}\right\|+\left\|w_{n}-z_{n}\right\| \\
& \leq\left\|T_{n} w_{n}-w_{n}\right\|+\beta_{n}\left\|z_{n}-w_{n}\right\|+\left\|w_{n}-z_{n}\right\| \\
& =\left(1+\left(1+\beta_{n}\right) \gamma_{n}\right)\left\|T_{n} w_{n}-w_{n}\right\| .
\end{aligned}
$$

These imply by Algorithm 1 that $\lim _{n \rightarrow+\infty}\left\|x_{n}-T_{n} x_{n}\right\|=0$ and $\lim _{n \rightarrow+\infty}\left\|y_{n}-z_{n}\right\|=0$. By Algorithm 1 and nonexpansivity of $T_{n}$, we have

$$
\begin{aligned}
&\left\|x_{n+1}-x_{n}\right\| \leq\left\|T_{n} z_{n}-x_{n}\right\|+\alpha_{n}\left\|T_{n} y_{n}-T_{n} z_{n}\right\| \\
& \leq\left\|T_{n} z_{n}-T_{n} x_{n}\right\|+\left\|T_{n} x_{n}-x_{n}\right\|+\alpha_{n}\left\|y_{n}-z_{n}\right\| \\
& \leq\left\|z_{n}-x_{n}\right\|+\left\|T_{n} x_{n}-x_{n}\right\|+\alpha_{n}\left\|y_{n}-z_{n}\right\| \\
& \leq\left\|z_{n}-w_{n}\right\|+\left\|w_{n}-x_{n}\right\|+\left\|T_{n} x_{n}-x_{n}\right\|+\alpha_{n}\left\|y_{n}-z_{n}\right\|, \\
&\left\|w_{n}-x_{n}\right\|=\theta_{n}\left\|x_{n}-x_{n-1}\right\| \rightarrow 0, \quad \text { and }\left\|z_{n}-w_{n}\right\|=\gamma_{n}\left\|T_{n} w_{n}-w_{n}\right\| \rightarrow 0 .
\end{aligned}
$$


These imply $\lim _{n \rightarrow+\infty}\left\|x_{n}-x_{n+1}\right\|=0$. Since $\left\{T_{n}\right\}$ satisfies the NST*-condition, we get $\omega_{w}\left(x_{n}\right) \subset$ $\Gamma:=\cap_{n=1}^{\infty}$ Fix $\left(T_{n}\right)$. Therefore, by Opial's lemma (Lemma 4 ), we conclude that $\left\{x_{n}\right\}$ converges weakly to a point in $\Gamma:=\cap_{n=1}^{\infty} \operatorname{Fix}\left(T_{n}\right)$. This completes the proof.

Finally, we apply our proposed algorithm, MWA, for solving the minimization problem (3) by setting $T_{n}=\operatorname{prox}_{c_{n} h}\left(I-c_{n} \nabla f\right)$, the forward-backward operator of $f$ and $h$ with respect to $c_{n}$, where $h: \mathbb{R}^{n} \rightarrow \mathbb{R} \cup\{+\infty\}$ is proper convex and lower semi-continuous, and $f: \mathbb{R}^{n} \rightarrow \mathbb{R}$ is a convex and differentiable function such that $\nabla f$ is a Lipschitz continuity with constant $L>0$.

By using the convergence result of Algorithm 1 (MWA) in Theorem 6, we obtain the convergence of Algorithm 2 (FBMWA) as in the following theorem.

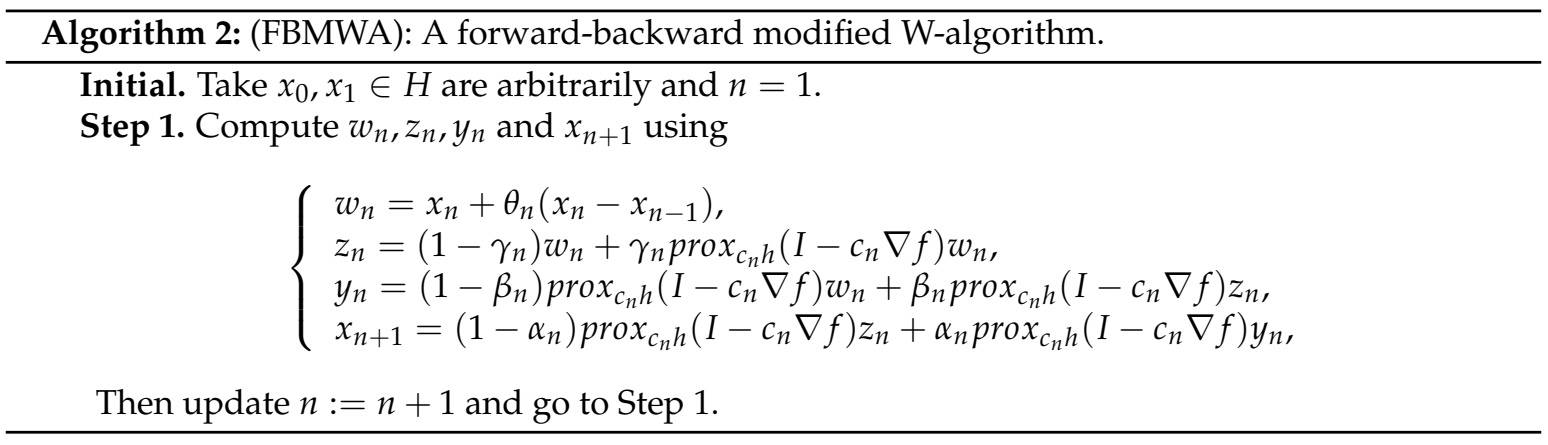

Theorem 7. Let $\left\{x_{n}\right\}$ be a sequence generated by Algorithm 2 (FBMWA) where $\gamma_{n}, \beta_{n}, \alpha_{n} \theta_{n}$ are the same as in Theorem 6 , and $c_{n} \in(0,2 / L)$ such that $\left\{c_{n}\right\}$ converges to $c$. Then the following holds

(i) $\left\|x_{n+1}-x^{*}\right\| \leq K \cdot \prod_{j=1}^{n}\left(1+2 \theta_{j}\right)$, where $K=\max \left\{\left\|x_{1}-x^{*}\right\|,\left\|x_{2}-x^{*}\right\|\right\}$ and $x^{*} \in \operatorname{Argmin}(f+$ h).

(ii) $\left\{x_{n}\right\}$ converges weakly to a point in $\operatorname{Argmin}(f+h)$.

Proof. Let $T$ be the forward-backward operator of $f$ and $h$ with respect to $c$, and $T_{n}$ be the forward-backward operator of $f$ and $h$ with respect to $c_{n}$, that is $T:=\operatorname{prox}_{c h}(I-c \nabla f)$ and $T_{n}:=\operatorname{prox}_{c_{n} h}\left(I-c_{n} \nabla f\right)$. Then $T$ and $\left\{T_{n}\right\}$ are nonexpansive operators for all $n$, and Fix $(T)=$ $\cap_{n=1}^{\infty} \operatorname{Fix}\left(T_{n}\right)=\operatorname{Argmin}(f+h)$; see Proposition 26.1 in [41]. By Lemma 1, we have that $\left\{T_{n}\right\}$ satisfies the NST* ${ }^{*}$-condition. Therefore, we obtain the required result directly by Theorem 6.

\section{Simulated Results for the Image Restoration Problem}

In this section, we apply Algorithm 2 (FBMWA) to solving the image restoration problem (2) and compare the deblurring efficiency of the FBMWA algorithm with FBS [30], IFBS [35], FISTA [28] and NAGA [37]. Our programs were written in Matlab and all algorithms ran on a laptop, Intel core i5, 4.00 GB RAM. All algorithms were applied to solving problem (2), where $f(y)=\|A y-a\|_{2}^{2}, h(y)=\lambda\|y\|_{1}$, $A$ is the blurring operator, $a$ is the observed image and $\lambda$ is the regularization parameter.

In this experiment, two gray-scale images, Lenna and Cameraman of size $256^{2}$ are considered the original images. The images went through a Gaussian blur of size $9^{2}$ and standard deviation $\sigma=4$. We use the peak signal-to-noise ratio (PSNR) [47] to measure the performance our the algorithms where $\operatorname{PSNR}\left(x_{n}\right)$ is defined by

$$
\operatorname{PSNR}\left(x_{n}\right)=10 \log _{10}\left(\frac{255^{2}}{M S E}\right)
$$


where $M S E=\frac{1}{M}\left\|x_{n}-\bar{x}\right\|_{2}^{2}, M$ is the number of image samples and $\bar{x}$ is the original image. It is noted that a higher value of PSNR of the same number of iteration shows a higher quality of deblurring image. The relative error is defined by

$$
\frac{\left\|x_{n}-x_{n-1}\right\|_{2}}{\left\|x_{n-1}\right\|_{2}} \leq \text { tol }
$$

where tol denotes a prescribed tolerance value. For these experiments, the regularization parameter was chosen to be $\lambda=5 \times 10^{-5}$, and the initial image was the blurred image. The Lipschitz constant $L$, was computed by the maximum eigenvalues of the matrix $A^{T} A$. We set parameters as follows:

$$
\begin{gathered}
\alpha_{n}=\beta_{n}=\gamma_{n}=0.5, c_{n}=\frac{n}{L(n+1)}, c=\frac{1}{L}, \theta_{n} \text { defined by (6) (for NAGA), } \\
\theta_{n}=\left\{\begin{array}{ll}
\frac{1}{n^{2}\left\|x_{n}-x_{n-1}\right\|_{2}^{2}} & \text { if } x_{n} \neq x_{n-1}, \\
0 & \text { otherwise, }
\end{array}\right. \text { (for IFBS), }
\end{gathered}
$$

and

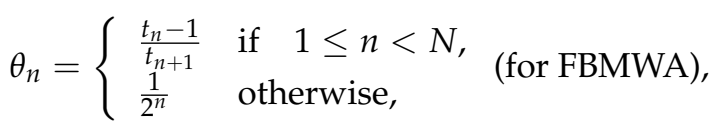

where $t_{n}$ is a sequence defined by $t_{1}=1$ and $t_{n+1}=\frac{1+\sqrt{1+4 t_{n}^{2}}}{2}$, and $N$ is a number of iterations that we want to stop. The results of deblurring image of Cameraman and Lenna with $1000^{\text {th }}$ iteration of the studied algorithms are shown in Table 1 and Figures 1 and 2.

Table 1. Comparison of image restorations of the studied methods.

\begin{tabular}{lcccc}
\hline & \multicolumn{2}{c}{ Cameraman } & \multicolumn{2}{c}{ Lenna } \\
\hline Algorithms & PSNR & Tol. & PSNR & Tol. \\
\hline FBS & 27.1953 & $2.32 \times 10^{-5}$ & 29.4907 & $1.73 \times 10^{-5}$ \\
IFBS & 27.1953 & $2.32 \times 10^{-5}$ & 29.4907 & $1.73 \times 10^{-5}$ \\
FISTA & 34.6659 & $4.13 \times 10^{-5}$ & 36.9324 & $3.34 \times 10^{-5}$ \\
NAGA & 35.6670 & $4.15 \times 10^{-5}$ & 37.8088 & $3.32 \times 10^{-5}$ \\
FBMWA & 36.2783 & $4.21 \times 10^{-5}$ & 38.2989 & $3.31 \times 10^{-5}$ \\
\hline
\end{tabular}
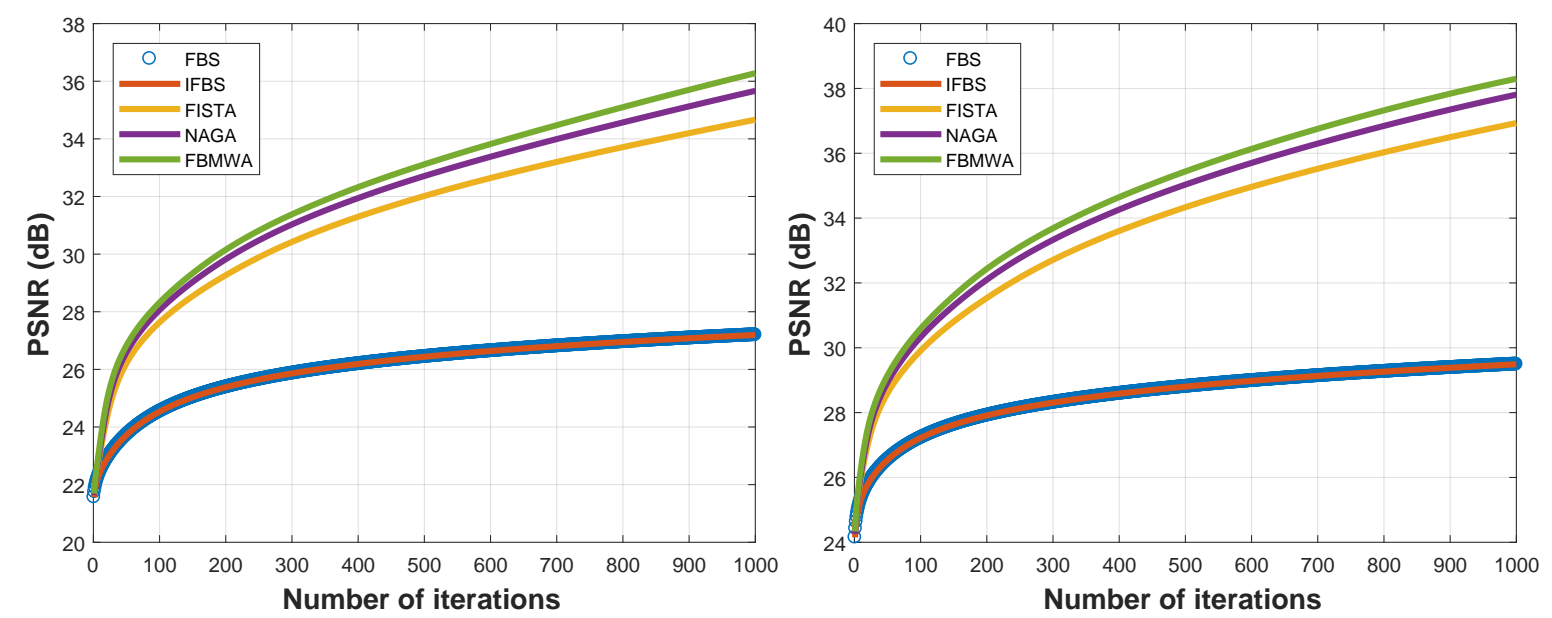

Figure 1. The graphs of peak signal-to-noise ratio (PSNR) for Cameraman (left) and Lenna (right). 
From Table 1 and the graph of PSNR in Figure 1, we see that FBMWA gives a higher PSNR than the other algorithms, so the performance of the image restoration of FBMWA is better than those of FBS, IFBS, FISTA and NAGA. We also see that after 1000 iterations, FBMWA gives a better result of deblurring for Cameraman and Lenna, as shown in Figure 2.

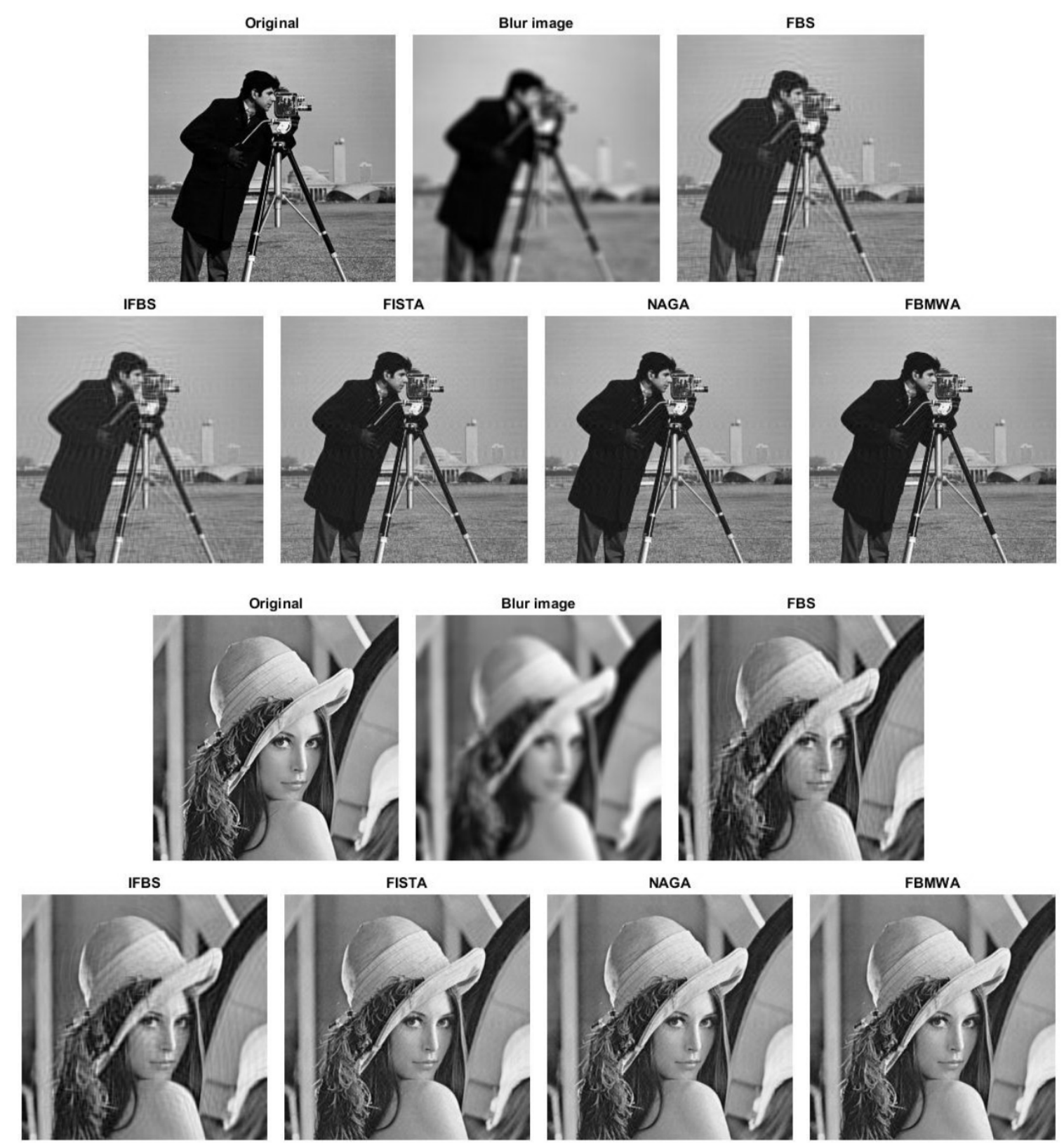

Figure 2. Results for deblurring of the Cameraman and Lenna.

The results of deblurring image of Cameraman and Lenna for the 1000th iteration of the FBMWA under different parameters $\theta_{n}$ are shown in Table 2 and Figures 3 and 4 , where $\theta_{n}$ is defined by

$$
\theta_{n}= \begin{cases}\mu_{n} & \text { if } 1 \leq n<N \\ \frac{1}{2^{n}} & \text { otherwise }\end{cases}
$$

where $\mu_{n}$ is a sequence of nonnegative real numbers and $N$ is a number of iterations that we want to stop. We observe that the inertial parameter $\theta_{n}$ using by FBMWA plays an important role in improving quality of deblurring image. It is noted that if $\left\{\theta_{n}\right\}$ is nondecreasing and tends to 1 , the values of PSNR 
increase, as shown in Table 2, Figure 3. However, we can see the result of the deblurring image of FBMWA with different inertial parameters $\theta_{n}$ (seven cases), as shown in Figure 4 . We also observe from Table 2 that the parameter $\mu_{n}=\frac{n}{n+1}$ gives a higher PSNR than the others.

Table 2. Effective parameters of our method for image restoration.

\begin{tabular}{cccccc}
\hline & & \multicolumn{2}{c}{ Cameraman } & \multicolumn{2}{c}{ Lenna } \\
\hline Case & Parameters & PSNR & Tol. & PSNR & Tol. \\
\hline 1 & $\mu_{n}=\frac{1}{2^{n}}$ & 27.8911 & $2.13 \times 10^{-5}$ & 30.1603 & $1.66 \times 10^{-5}$ \\
\hline 2 & $\mu_{n}=\frac{10}{n^{2}}$ & 27.9003 & $2.12 \times 10^{-5}$ & 30.1693 & $1.65 \times 10^{-5}$ \\
\hline 3 & $\mu_{n}=0.5$ & 28.7146 & $2.00 \times 10^{-5}$ & 30.9771 & $1.60 \times 10^{-5}$ \\
\hline 4 & $\mu_{n}=0.9$ & 30.9920 & $1.81 \times 10^{-5}$ & 33.2838 & $1.47 \times 10^{-5}$ \\
\hline 5 & $\mu_{n}=\frac{t_{n}-1}{t_{n+1}}, t_{1}=1$, & 36.2783 & $4.21 \times 10^{-5}$ & 38.2989 & $3.31 \times 10^{-5}$ \\
\hline 6 & $t_{n+1}=\frac{1+\sqrt{1+4 t_{n}^{2}}}{2}$, & & & & \\
\hline 7 & $\mu_{n}=\frac{n}{n+1}$ & 37.0979 & $1.63 \times 10^{-4}$ & 38.8562 & $1.30 \times 10^{-4}$ \\
\hline & $\mu_{n}=1$ & 30.6832 & $9.13 \times 10^{-4}$ & 32.7996 & $7.07 \times 10^{-4}$ \\
\hline
\end{tabular}
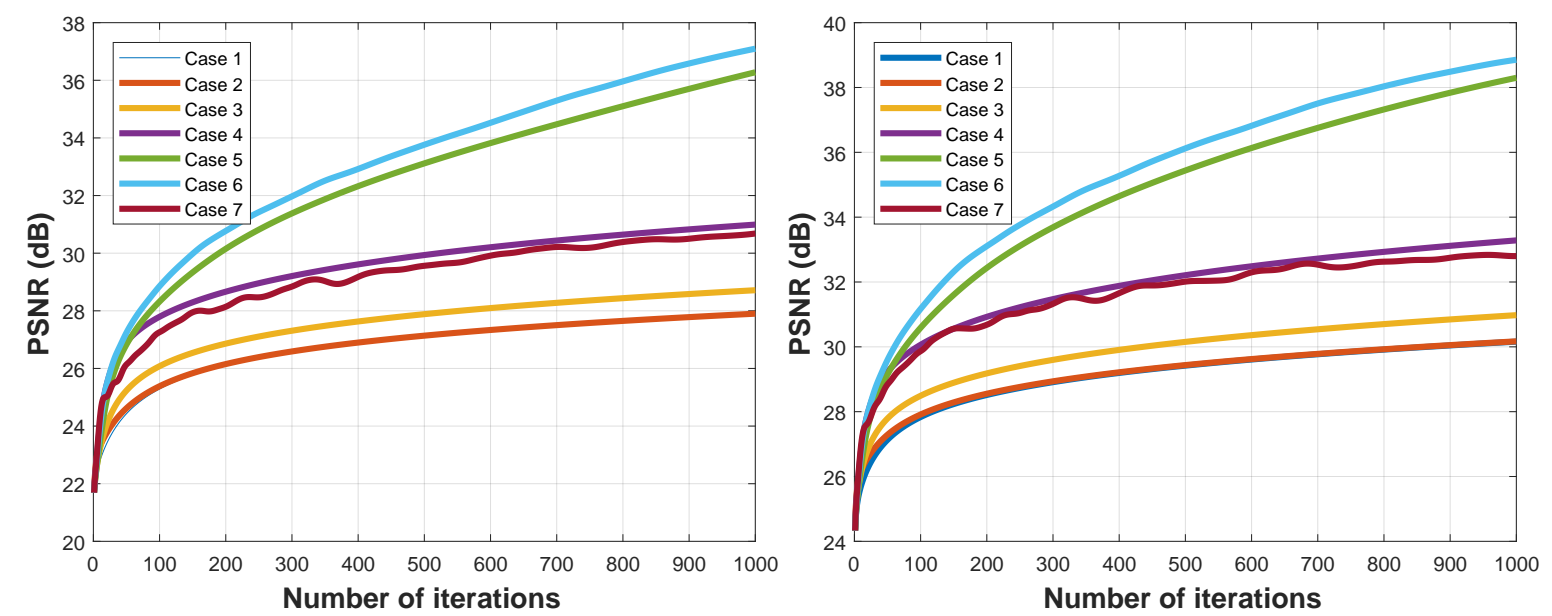

Figure 3. The graphs of PSNR of the FBMWA under different parameters $\theta_{n}$ for Cameraman (left) and Lenna (right).

Open problem: It is noted that we can choose $\theta_{n}$ as in (21) for the Algorithm 2, and convergence of Algorithm 2 can be guaranteed by Theorem 7. Can we use $\theta_{n}$ as defined by (6) for Algorithm 2?

\section{Conclusions}

In this work, we proposed a modified W-algorithm for solving a common fixed point problem of a family of nonexpansive operators and proved the weak convergence result of the proposed method under some control conditions. We applied our main result to solving a minimization problem in the form of the sum of two proper lower semi-continuous and convex functions. As applications, we applied our algorithm, FBMWA, to solving image restoration problems. Moreover, we did some numerical experiments to illustrate the performance of the studied algorithms and show that PSNR of FBMWA is better than those of the FBS [30], IFBS [35], FISTA [28] and NAGA [37]. 


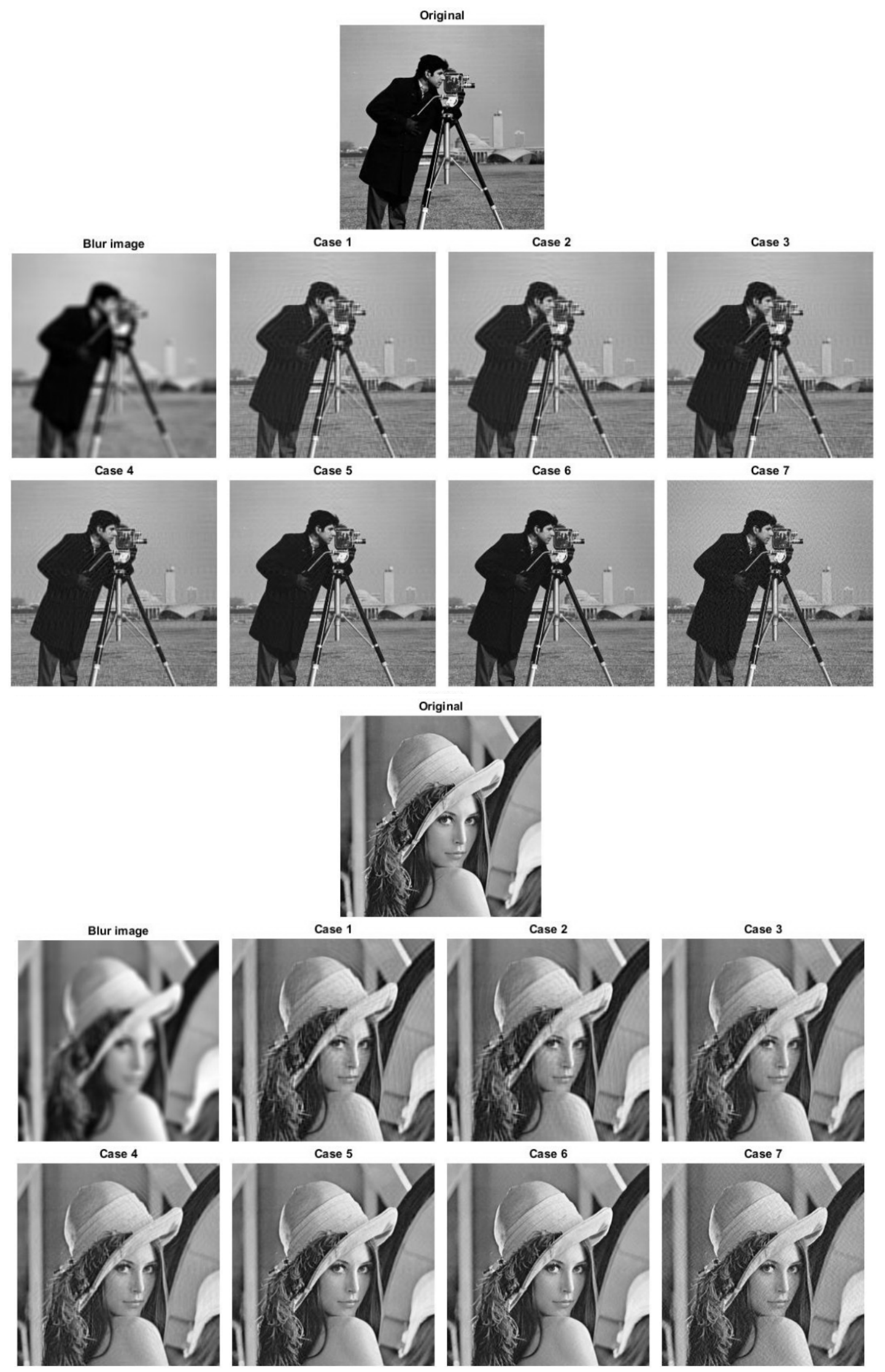

Figure 4. Results of FBMWA for deblurring of the Cameraman and Lenna. 
Author Contributions: Funding acquisition and supervision, S.S.; writing-review and editing and software, A.H. All authors have read and agreed to the published version of the manuscript.

Funding: This work was funded by Thailand Science Research and Innovation under the project IRN62W0007 and Chiang Mai University.

Acknowledgments: This work was supported by Thailand Science Research and Innovation under the project IRN62W0007. The opinions in the research report belong to the researchers; Thailand Science Research and Innovation do not always have to agree. The second author would also like to thank Chiang Mai University for the financial support.

Conflicts of Interest: The authors declare no conflict of interest.

\section{References}

1. Bauschke, H.H. The approximation of fixed points of compositions of nonexpansive mappings in Hilbert space. J. Math. Anal. Appl. 1996, 202, 150-159. [CrossRef]

2. Chidume, C.E.; Bashir, A. Convergence of path and iterative method for families of nonexpansive mappings. Appl. Anal. 2008, 67, 117-129. [CrossRef]

3. Halpern, B. Fixed points of nonexpansive maps. Bull. Am. Math. Soc. 1967, 73, 957-961. [CrossRef]

4. Ishikawa, S. Fixed points by a new iteration method. Proc. Am. Math. Soc. 1974, 44, 147-150. [CrossRef]

5. Klen, R.; Manojlović, V.; Simić, S.; Vuorinen, M. Bernoulli inequality and hypergeometric functions. Proc. Am. Math. Soc. 2014, 142, 559-573. [CrossRef]

6. Kunze, H.; La Torre, D.; Mendivil, F.; Vrscay,E.R. Generalized fractal ntransforms and self-similar objects in cone metric spaces. Comut. Math. Appl. 2012, 64, 1761-1769. [CrossRef]

7. Mann, W.R. Mean value methods in iteration. Proc. Am. Math. Soc. 1953, 4, 506-510. [CrossRef]

8. Radenović, S.; Rhoades, B.E. Fixed point theorem for two non-self mappings in cone metric spaces. Comput. Math. Appl. 2009, 57, 1701-1707. [CrossRef]

9. Todorcević, V. Harmonic Quasiconformal Mappings and Hyperbolic Type Metrics; Springer Nature Switzerland AG: Basel, Switzerland, 2019.

10. Byrne, C. Iterative oblique projection onto convex subsets and the split feasibility problem. Inverse Probl. 2002, 18, 441-453. [CrossRef]

11. Byrne, C. Aunified treatment of some iterative algorithms in signal processing and image reconstruction. Inverse Probl. 2004, 20, 103-120. [CrossRef]

12. Cholamjiak, P.; Shehu, Y. Inertial forward-backward splitting method in Banach spaces with application to compressed sensing. Appl. Math. 2019, 64, 409-435. [CrossRef]

13. Combettes, P.L.; Wajs, V. Signal recovery by proximal forward-backward splitting. Multiscale Model. Simul. 2005, 4, 1168-1200. [CrossRef]

14. Kunrada, K.; Pholasa, N.; Cholamjiak, P. On convergence and complexity of the modified forward-backward method involving new linesearches for convex minimization. Math. Meth. Appl. Sci. 2019, 42, 1352-1362.

15. Suantai, S.; Eiamniran, N.; Pholasa, N.; Cholamjiak, P. Three-step projective methods for solving the split feasibility problems. Mathematics 2019, 7, 712. [CrossRef]

16. Suantai, S.; Kesornprom, S.; Cholamjiak, P. Modified proximal algorithms for finding solutions of the split variational inclusions. Mathematics 2019, 7, 708. [CrossRef]

17. Thong, D.V.; Cholamjiak, P. Strong convergence of a forward-backward splitting method with a new step size for solving monotone inclusions. Comput. Appl. Math. 2019, 38. [CrossRef]

18. Censor, Y.; Bortfeld, T.; Martin, B.; Trofimov, A. A unified approach for inversion problems in intensity-modulated radiation therapy. Phys. Med. Biol. 2006, 51, 2353-2365. [CrossRef]

19. Censor, Y.; Elfving, T.; Kopf, N.; Bortfeld, T. The multiple set split feasibility problem and its applications. Inverse Probl. 2005, 21, 2071-2084. [CrossRef]

20. Censor, Y.; Motova, A.; Segal, A. Perturbed projections and subgradient projections for the multiple-sets feasibility problem. J. Math. Anal. 2007, 327, 1244-1256. [CrossRef]

21. Phuengrattana, W.; Suantai, S. On the rate of convergence of Mann, Ishikawa, Noor and SP-iterations for continuousfunctions on an arbitrary interval. J. Comput. Appl. Math. 2011, 235, 3006-3014. [CrossRef] 
22. Wongyai, S.; Suantai, S. Convergence Theorem and Rate of Convergence of a New Iterative Method for Continuous Functions on Closed Interval. In Proceedings of the AMM and APAM Conference Proceedings, Bankok, Thailand, 23-25 May 2016; pp. 111-118.

23. Ben-Tal, A.; Nemirovski, A. Lectures on Modern Convex Optimization, Analysis, Algorithms, and Engineering Applications; MPS/SIAM Ser. Optim.; SIAM: Philadelphia, PA, USA, 2001.

24. Bioucas-Dias, J.; Figueiredo, M. A new TwIST: Two-step iterative shrinkage/thresholding algorithms for image restoration. IEEE Trans. Image Process. 2007, 16, 2992-3004. [CrossRef] [PubMed]

25. Chen, S.S.; Donoho, D.L.; Saunders, M.A. Atomic decomposition by basis pursuit. SIAM J. Sci. Comput. 1998, 20, 33-61. [CrossRef]

26. Donoho, D.L.; Johnstone, I.M. Adapting to unknown smoothness via wavelet shrinkage. J. Am. Statist. Assoc. 1995, 90, 1200-1224. [CrossRef]

27. Figueiredo, M.A.T.; Nowak, R.D.; Wright, S.J. Gradient projection for sparse reconstruction: Application to compressed sensing and other inverse problems. IEEE J. Sel. Top. Signal Process. 2007, 1, 586-597. [CrossRef]

28. Beck, A.; Teboulle, M. A fast iterative shrinkage-thresholding algorithm for linear inverse problems. SIAM J. Imaging Sci. 2009, 2, 183-202. [CrossRef]

29. Tibshirani, R. Regression shrinkage and selection via the lasso. J. R. Stat. Soc. B Methodol. 1996, 58, $267-288$. [CrossRef]

30. Lions, P.L.; Mercier, B. Splitting algorithms for the sum of two nonlinear operators. SIAM J. Numer. Anal. 1979, 16, 964-979. [CrossRef]

31. Moreau, J.J. Proximité et dualité dans un espace hilbertien. Bull. Soc. Math. Fr. 1965, 93, 273-299. [CrossRef]

32. Figueiredo, M.A.T.; Nowak, R.D. An EM algorithm for wavelet-based image restoration. IEEE Trans. Image Process. 2003, 12, 906-916. [CrossRef]

33. Daubechies, I.; Defrise, M.; Mol, C.D. An iterative thresholding algorithm for linear inverse problems with a sparsity constraint. Commun. Pure Appl. Math. 2004, 57, 1413-1457. [CrossRef]

34. Hale, E.T.; Yin, W.; Zhang, Y. A Fixed-Point Continuation Method for 11-Regularized Minimization. Siam J. Optim. 2008, 19, 1107-1130. [CrossRef]

35. Moudafi, A.; Oliny, M. Convergence of a splitting inertial proximal method for monotone operators. J. Comput. Appl. Math. 2003, 155, 447-454. [CrossRef]

36. Liang, J.; Schonlieb, C.B. Improving fista: Faster, smarter and greedier. arXiv 2018, arXiv:1811.01430.

37. Verma, M.; Shukla, K.K. A new accelerated proximal gradient technique for regularized multitask learning framework. Pattern Recogn. Lett. 2017, 95, 98-103. [CrossRef]

38. Nakajo, K.; Shimoji, K.; Takahashi, W. Strong convergence to common fixed points of families of nonexpansive mappings in Banach spaces. J. Nonlinear Convex Anal. 2007, 8, 11-34.

39. Nakajo, K.; Shimoji, K.; Takahashi, W. On strong convergence by the hybrid method for families of mappings in Hilbert spaces. Nonlinear Anal. Theor. Methods Appl. 2009, 71, 112-119. [CrossRef]

40. Boyd, S.; Vandenberghe, L. Convex Optimization; Cambridge University Press: New York, NY, USA, 2004.

41. Bauschke, H.H.; Combettes, P.L. Convex Analysis and Monotone Operator Theory in Hilbert Spaces; Springer: New York, NY, USA, 2011.

42. Burachik, R.S.; Iusem, A.N. Set-Valued Mappings and Enlargements of Monotone Operator; Springer Science Business Media: New York, NY, USA, 2007.

43. Bussaban, L.; Suantai, S.; Kaewkhao, A. A parallel inertial S-iteration forward-backward algorithm for regression and classification problems. Carpathian J. Math. 2020, 36, 21-30.

44. Takahashi, W. Introduction to Nonlinear and Convex Analysis; Yokohama Publishers: Yokohama, Japan, 2009.

45. Tan, K.; Xu, H.K. Approximating fixed points of nonexpansive mappings by the ishikawa iteration process. J. Math. Anal. Appl. 1993, 178, 301-308. [CrossRef]

46. Moudafi, A.; Al-Shemas, E. Simultaneous iterative methods for split equality problem. Trans. Math. Program. Appl. 2013, 1, 1-11.

47. Thung, K.; Raveendran, P. A survey of image quality measures. In Proceedings of the International Conference for Technical Postgraduates (TECHPOS), Kuala Lumpur, Malaysia, 14-15 December 2009; pp. 1-4.

(C) 2020 by the authors. Licensee MDPI, Basel, Switzerland. This article is an open access article distributed under the terms and conditions of the Creative Commons Attribution (CC BY) license (http:/ / creativecommons.org/licenses/by/4.0/). 